\title{
Checklist of dragonflies and damselflies (Insecta: Odonata) of the Amazonas state, Brazil
}

\author{
Ricardo Koroiva ${ }^{1 *}$, Ulisses Gaspar Neiss ${ }^{1,2}$, Günther Fleck ${ }^{3}$ \& Neusa Hamada ${ }^{1}$ \\ ${ }^{1}$ Instituto Nacional de Pesquisas da Amazônia, Laboratório de Citotaxonomia e Insetos Aquáticos, Manaus, \\ AM, Brasil \\ ${ }^{2}$ Instituto de Criminalística, Departamento de Polícia Técnica-Cientifica, Manaus, AM, Brasil \\ ${ }^{3}$ Autonomous researcher, Lagorce 07150, France \\ *Corresponding author: Ricardo Koroiva, e-mail: ricardo.koroiva@gmail.com
}

KOROIVA, R., NEISS, U. G., FLECK, G., HAMADA, N. Checklist of dragonflies and damselflies(Insecta: Odonata) of the Amazonas state, Brazil. Biota Neotropica. 20(1): e20190877. http://dx.doi.org/10.1590/1676-0611-BN-2019-0877

\begin{abstract}
Here we provide a checklist of the odonates from Amazonas state, Brazil. We registered 324 species and 101 genera, making Amazonas the Brazilian state with the most Odonata species recorded. The families with the highest number of species were Coenagrionidae with 32 genera and 101 species, followed by Libellulidae with 28 genera and 100 species and Gomphidae with 12 genera and 45 species. Some regions of Amazonas state remain poorly explored, such as the southern area, and large municipalities, such as São Gabriel da Cachoeira. This work underlines the importance of the biological diversity from Amazonas state and the Amazonian Biome for Odonata species richness in Brazil and shows that many areas in the world's largest tropical forest have not yet been sampled.
\end{abstract}

Keywords: Aquatic insects, List of species, Anisoptera, Zygoptera, Amazon, Neotropical region.

\section{Checklist das libélulas (Insecta: Odonata) do estado do Amazonas, Brasil}

Resumo: Aqui nós apresentamos a lista de espécies de libélulas presentes no estado do Amazonas, Brasil. Nós registramos 324 espécies e 101 gêneros, tornando o estado com o maior número de espécies de libélulas registradas no país. As famílias com maior número de espécies foram Coenagrionidae, com 32 gêneros e 101 espécies, seguido por Libellulidae, com 28 gêneros e 100 espécies e Gomphidae com 12 gêneros e 45 espécies. Regiões do estado do Amazonas permanecem pouco exploradas, como a parte sul e os municípios com grande extensão territorial, como São Gabriel da Cachoeira. Este trabalho reconhece a importância da diversidade biológica do estado do Amazonas e do bioma Amazônico para a riqueza de espécies de Odonata no Brasil e mostra que ainda existem muitas áreas não amostradas na maior floresta tropical do mundo.

Palavras-chave: Insetos aquáticos, lista de espécies, Anisoptera, Zygoptera, Amazônia, região Neotropical. 


\section{Introduction}

Considered one of the most diverse regions for Odonata, the Neotropical region has more than 1,700 species, and in Brazil 749 species are registered (Olaya 2019). Dragonflies and damselflies (Insecta: Odonata) constitute an important group of aquatic insects characterized by their extensive predation, with larvae being important links for fish and other aquatic vertebrates in food webs. Besides their ecological importance, odonates have great artistic appeal, and have been used in environmental quality assessments using several environmental quality metrics (Abbott 2015).

Even with such importance, the taxonomic knowledge about Odonata in Brazil is still heterogeneous. As evidenced by Dalzochio et al. (2018), Brazilian studies concerning Odonata fauna are mainly concentrated in the south-center of Brazil, with species lists registered for the states of São Paulo (Costa et al. 2000), Rio de Janeiro (Costa \& Santos 2000), Mato Grosso do Sul (Koroiva et al. 2017; Rodrigues et al. 2018), Rio Grande do Sul (Dalzochio et al. 2018; Pires et al. 2019), Espírito Santo (Costa \& Oldrini 2005), Goiás and the Federal District (Nóbrega \& De Marco 2011). Despite the increasing number of new species descriptions, the lack of species lists in other regions and states make the analysis of current sampling distributions and knowledge about Odonata species distribution inviable, which directly affects large-scale conservation assessments (Silveira et al. 2010).

The Amazonas state located in the northern region of Brazil, presents the largest territorial extension and stands out (IBGE 2019) as it encompasses Amazonian and Cerrado biomes (Parédio 2012). In recent years, political issues and increased deforestation rates in both biomes have raised concerns for the maintenance of their forested areas and associated fauna (Rochedo et al. 2018, Fearnside 2019). Regarding Odonata, several studies have been dedicated to increasing knowledge about its fauna in Amazonas state in the last 120 years, however, almost all of them are restricted to taxonomic descriptions and ecological analysis and do not provide information about the total number of species. In the most recent estimate, Neiss \& Hamada (2014) indicated about 262 Odonata species in Amazonas, representing 35\% of the known species from Brazil. Considering this information gap in Amazonas, the main objective of this work was to present the diversity of Odonata species in this state, as well as their known locations in the municipalities.

\section{Materials and Methods}

\section{Study area}

The state of Amazonas has an area of $1,559,168 \mathrm{~km}^{2}$ (IBGE 2019) and stands out due to the predominant presence of the Amazonian Biome. The state shares borders with Pará, Mato Grosso, Rondônia, Acre and Roraima states and with the countries of Venezuela, Peru and Colombia (Figure 1). The geographical relief of Amazonas state is constituted by depressions (Western Amazon Depression, Northern Amazonian Depression and Southern Amazon Depression), plains, and plateaus (North-Amazonian Residual Plateau and Plateau of the Eastern Amazon). Its hydrology is formed mainly by the eastern basin of the Amazon River and is composed of most of the rivers from this hydrographic basin, whose tributaries are the rivers Negro, Japurá, Solimões, Juruá, Purus and Madeira. The northern and eastern regions of the state are located in the northern Amazonian plateau, which contain the highest altitudes (above 2,950 m) in Brazil: "Pico da Neblina" and "31 de Março" mountains (Parédio 2012). The vegetation types vary from Ombrophylous Forest to "Campinarana" and Savana in the Amazon and Cerrado biomes. According to Köppen's climatic classification, the predominant climate is Af type, tropical without dry season, except in the southern part of the state, which is considered Am, tropical monsoon. The average annual precipitation varies between $1,900 \mathrm{~mm}$ in the extreme southwest and above 3,000 $\mathrm{mm}$ in other areas and average temperatures are above $23^{\circ} \mathrm{C}$ (Alvares et al. 2013).

\section{Elaboration of the list}

To prepare the list, we gathered information from the Invertebrates Collection at the Instituto Nacional de Pesquisas da Amazônia (INPA), Manaus, Brazil. Additional data were collected from 102 publications, published between 1897 and 2019, the sites SpeciesLink (http://splink. cria.org.br/) and "Catálogo Taxonômico da Fauna do Brasil" (http:// fauna.jbrj.gov.br ; "Brazilian Fauna Taxonomic Catalog" in English; Pinto 2019) (see Table 1). We also gathered information about the municipalities where the species were collected (Table 1). For systematic classification, we followed Dijkstra et al. (2014, 2013) and Fleck (2018).

\section{Results}

The total number of Odonata species recorded in Amazonas is 324 (Table 2). Some species recorded in this study are shown in Figure 2. The total number of genera recorded for the state is 101, distributed in 16 families; this number include one genus from the Calopterygoidea "Incertae sedis group 3" and one genus from the Libelluloidea "incertae sedis". Coenagrionidae is the family with the highest number of records, with 32 genera and 101 species, followed by Libellulidae with 28 genera and 100 species, Gomphidae with 12 genera and 45 species, and Aeshnidae with eight genera and 27 species. The other families are: Calopterygidae (two genera, 12 species), Corduliidae (two genera, four species), Dicteriadidae (two genera, two species), Heteragrionidae (two genera, seven species), Lestidae (one genus, one species), Megapodagrionidae (one genus, one species), Perilestidae (two genera, seven species), Philogeniidae (one genus, two species), Platystictidae (one genus, one species), Polythoridae (three genera, 10 species), Rimanellidae (one genus, one species) and Oxygastridae sensu Fleck (2018) (one genus, one species). Calopterygoidea "incertae sedis group 3" was represented by one genus and one species and Libelluloidea incertae sedis was also represented by one genus and one species.

Considering the territorial divisions of the state, Manaus (code number 1) municipality has the most species registered, 163 species, followed by Presidente Figueiredo (code number 2), 100 species, and Barcelos (code number 4), 71 species. In addition, only 33 of the 62 municipalities in Amazonas have published Odonata records.

\section{Discussion}

The 324 species listed from Amazonas state represent about 43\% of the known species in Brazil (749 species). This number increases the species records for the state by $23 \%$ (Neiss \& Hamada 2014). With the results presented in this study, Amazonas state is the Brazilian state with the highest number of Odonata species recorded, followed by 


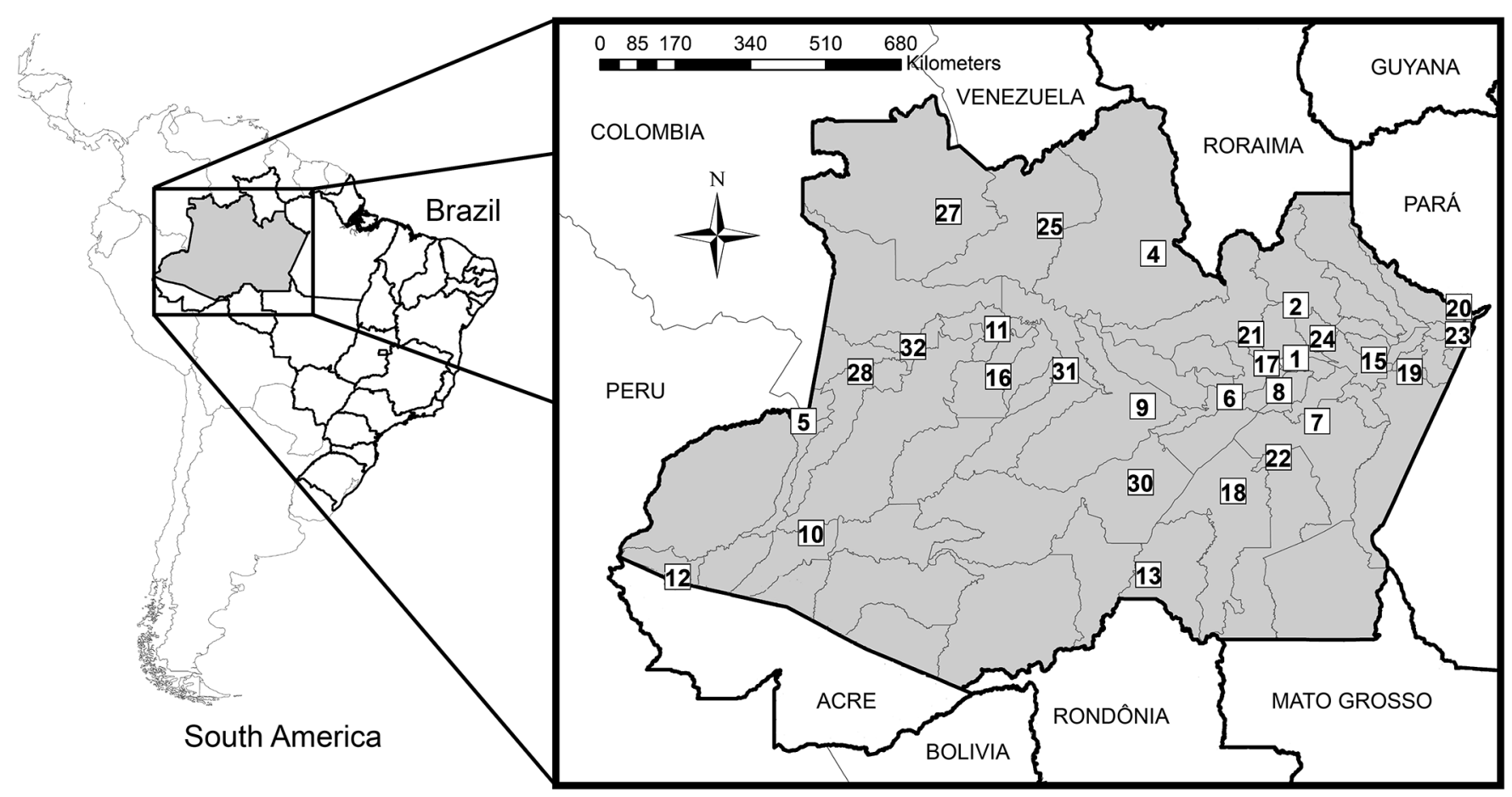

Figure 1. Left, Map of South America highlighting the geopolitical division of Brazil and Amazonas State (grey) (Datum: WGS 84); Right, Municipalities with Odonata recorded in Amazonas State. For code numbers see Table 1.

Table 1. Numbers and codes for municipalities and references used in the list of Odonata species from Amazonas state, Brazil. Where the municipality is unknown, only the state is provided.

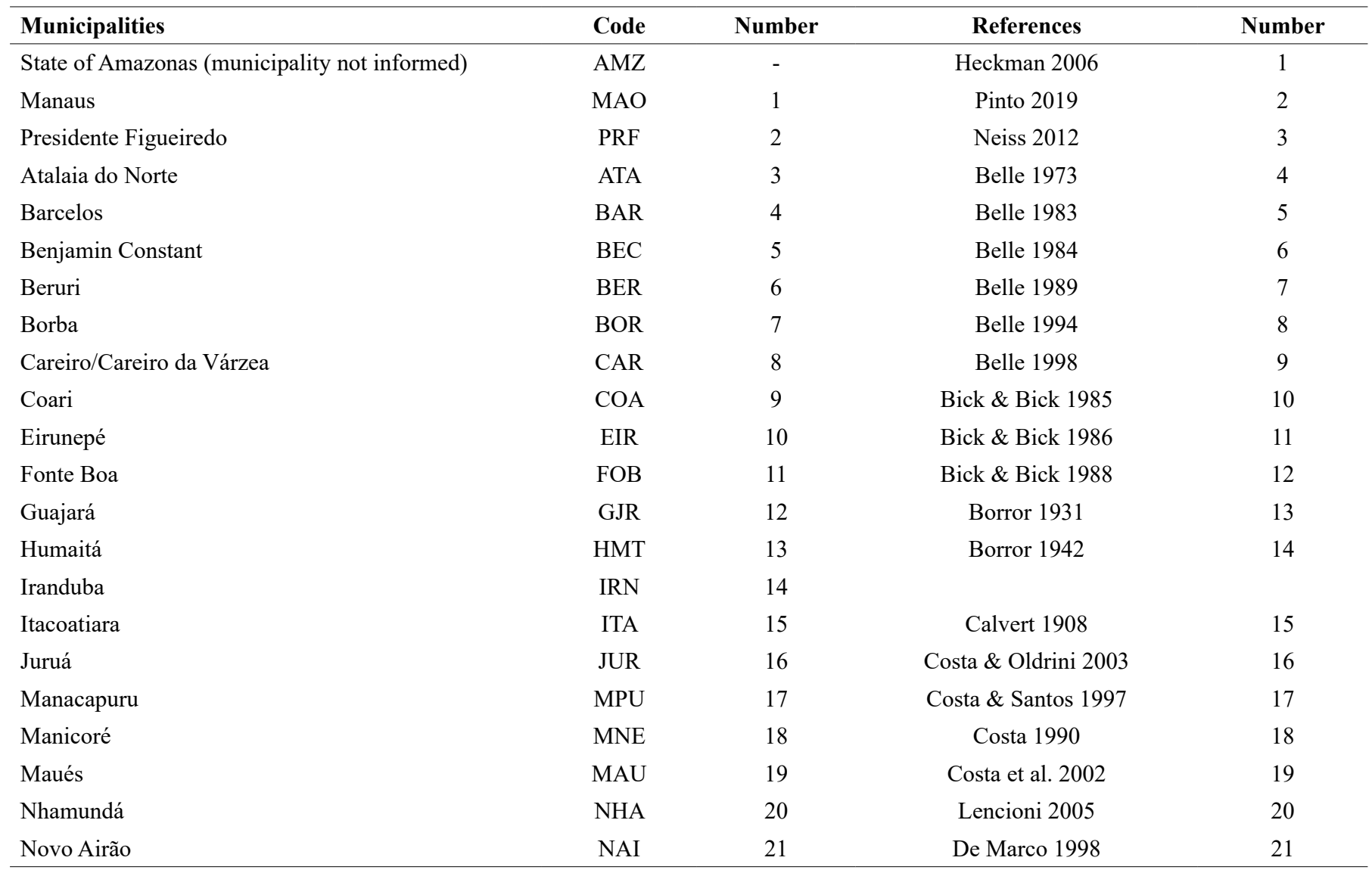


Koroiva R. et al.

Continuation...

\begin{tabular}{|c|c|c|c|c|}
\hline Municipalities & Code & Number & References & Number \\
\hline Novo Aripuanã & NRP & 22 & De Marmels \& Rácenis 1982 & 22 \\
\hline Parintins & PAR & 23 & De Marmels 1987 & 23 \\
\hline Santa Isabel do Rio Negro & SIR & 25 & De Marmels 2001 & 25 \\
\hline Santo Antônio do Içá & SAI & 26 & De Marmels \& Neiss 2011 & 26 \\
\hline Tabatinga & TAB & 29 & Fleck \& Neiss 2012 & 29 \\
\hline Tapauá & TAP & 30 & Fulan et al. 2015 & 30 \\
\hline Tefé & TEF & 31 & Garrison \& Ellenrieder 2006 & 31 \\
\hline \multirow[t]{27}{*}{ Tonantins } & TON & 32 & Garrison \& Ellenrieder 2009 & 32 \\
\hline & & & Garrison 2004 & 36 \\
\hline & & & Garrison 2006 & 37 \\
\hline & & & Garrison 2009 & 38 \\
\hline & & & Garrison 2014 & 39 \\
\hline & & & Geijskes 1963 & 40 \\
\hline & & & Geijskes 1970 & 41 \\
\hline & & & Geijskes 1984 & 42 \\
\hline & & & Guillermo-Ferreira et al. 2014 & 43 \\
\hline & & & Hamada \& Oliveira 2003 & 44 \\
\hline & & & Machado 2002 & 50 \\
\hline & & & Machado 2007 & 51 \\
\hline & & & Machado 2009a & 52 \\
\hline & & & Machado 2009b & 53 \\
\hline & & & Machado 2012 & 54 \\
\hline & & & Machet 1990 & 55 \\
\hline & & & May 1991 & 56 \\
\hline & & & Monteiro-Júnior et al. 2013 & 57 \\
\hline & & & Montgomery 1940 & 58 \\
\hline & & & Neiss \& Hamada 2010 & 59 \\
\hline & & & Neiss \& Hamada 2016 & 60 \\
\hline & & & Neiss et al. 2008 & 61 \\
\hline & & & Neiss et al. 2013 & 62 \\
\hline & & & Pessacq 2014 & 63 \\
\hline & & & Pessacq et al. 2012 & 64 \\
\hline & & & Pinto \& Carvalho 2010 & 65 \\
\hline & & & Pinto \& Lamas 2010 & 66 \\
\hline
\end{tabular}


Checklist of odonates from Amazonas state, Brazil

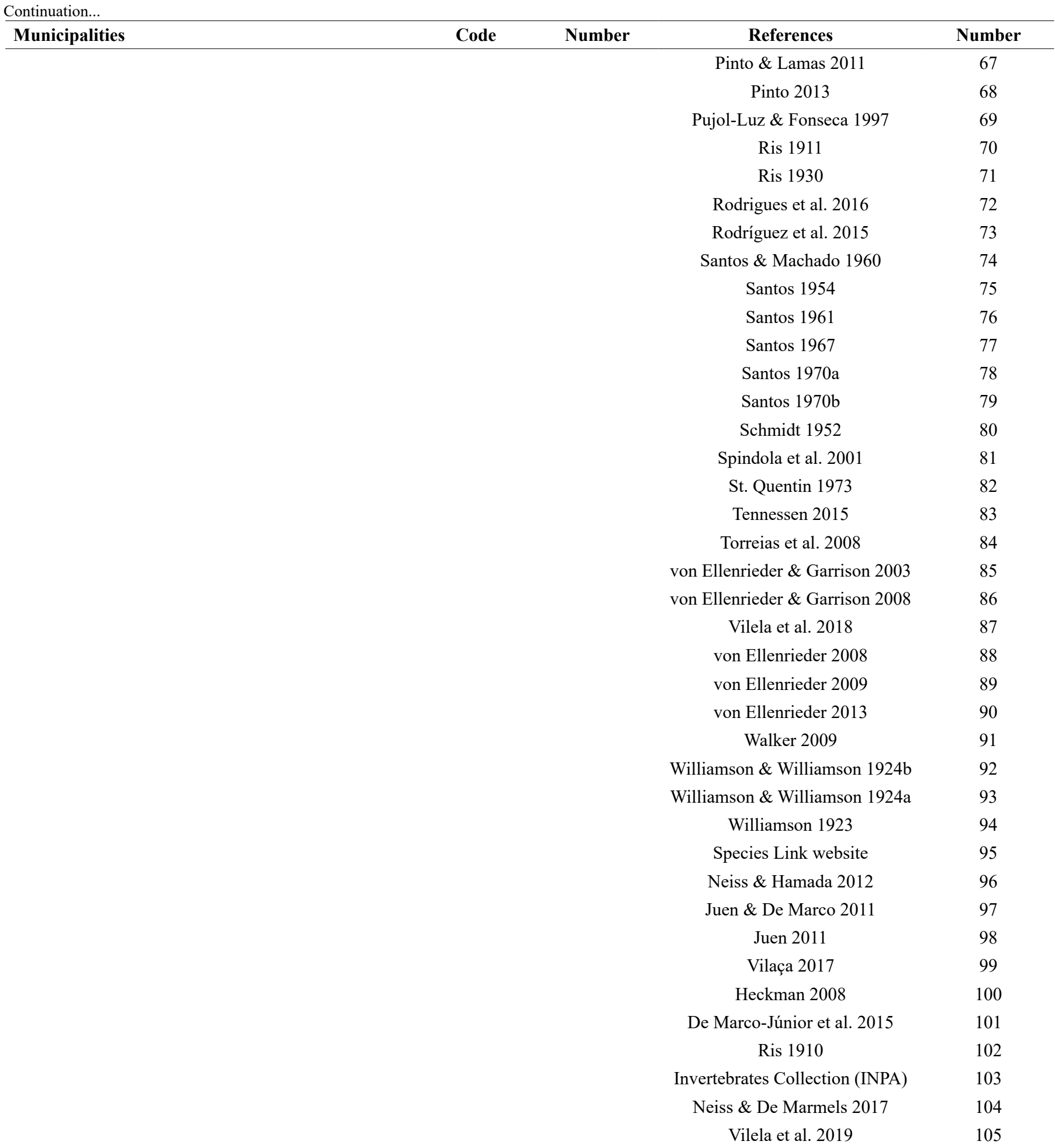

Rio de Janeiro, 280 species (Costa \& Santos 2000), Minas Gerais, 269 species (Vilela et al. 2020), São Paulo, 251 species (Costa et al. 2000), Mato Grosso do Sul, 209 species (Koroiva et al. 2017; Rodrigues et al. 2018), Rio Grande do Sul, 183 species (Pires et al. 2019), Espírito Santo, 180 species (Costa \& Oldrini 2005), and Goiás and the Federal District, 152 species (Nóbrega \& De Marco 2011).

We highlight that five species were not included in the results due to imprecise information. Despite being cited for Amazonas state in
Heckman (2008), in the original description of Planiplax machadoi Santos, 1949, Santos (1949) states that the species is present in "Amazonas", but in the text the author explains that specimens came from Pará state. Similar problem occurs with Erythrodiplax juliana Ris, 1911 and Erythrodiplax latimaculata Ris, 1911. Borror (1942) states that these species are present in Amazonas state; however, the specimens were collected in Monte Roraima and Uraricoera, respectively, which currently belong to Roraima state. Finally, 
Koroiva R. et al.

Table 2. Odonata species recorded for Amazonas State, Brazil. For references and municipalities codes, please check Table 1.

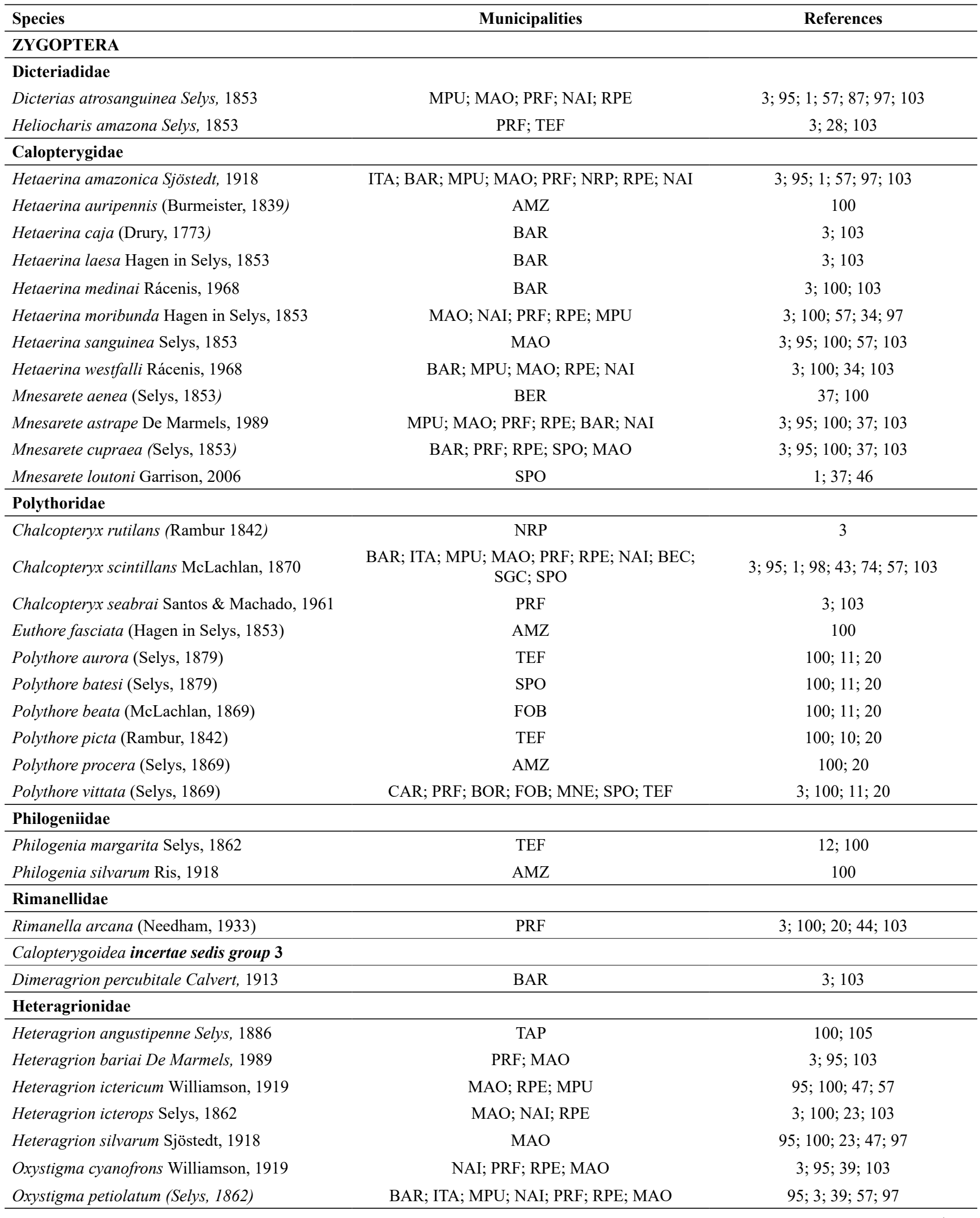


Checklist of odonates from Amazonas state, Brazil

Continuation...

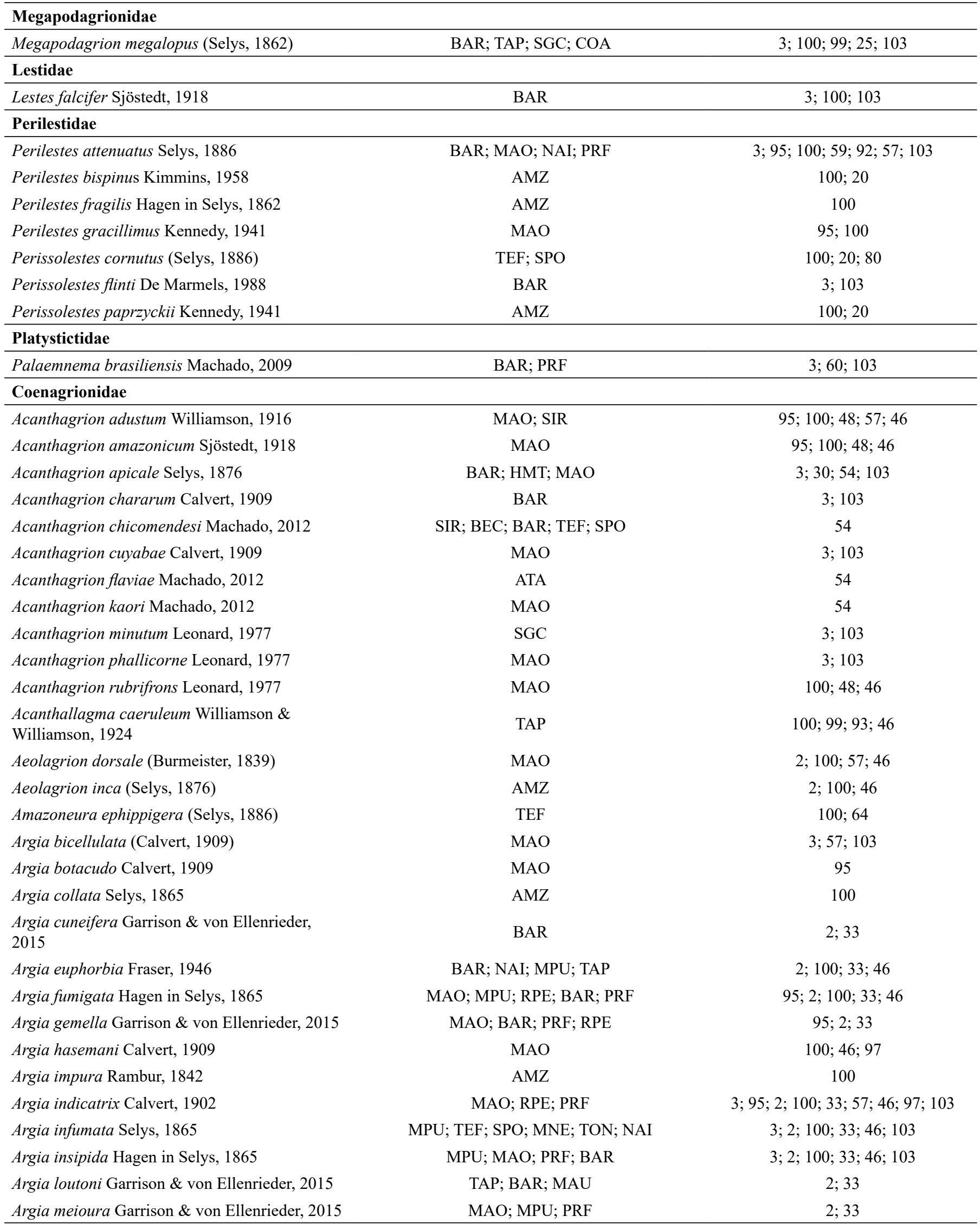


Continuation...

\begin{tabular}{|c|c|c|}
\hline Argia mollis Hagen in Selys, 1865 & $\mathrm{AMZ}$ & $100 ; 46$ \\
\hline Argia oculata Hagen in Selys, 1865 & BAR; PRF; NAI; MAO & $2 ; 100 ; 33 ; 46 ; 97$ \\
\hline Argia palmata Garrison \& von Ellenrieder, 2015 & MAO & $2 ; 33$ \\
\hline Argia reclusa Selys, 1865 & AMZ & $100 ; 46$ \\
\hline Argia subapicalis Calvert, 1909 & MAO & 97 \\
\hline Argia translata Hagen in Selys, 1865 & $\mathrm{AMZ}$ & 2 \\
\hline Bromeliagrion rehni Garrison, 2005 & MAO & $3 ; 95 ; 100 ; 84 ; 46 ; 103$ \\
\hline Calvertagrion charis Tennessen, 2015 & AMZ & $2 ; 83$ \\
\hline Calvertagrion minutissimum (Selys, 1876) & AMZ & $2 ; 100 ; 46$ \\
\hline Epipleoneura capilliformis (Selys, 1886) & MPU; MAO; NAI; PRF; RPE & $3 ; 95 ; 63 ; 57 ; 103$ \\
\hline Epipleoneura fuscaenea Williamson, 1915 & NRP & $3 ; 103$ \\
\hline Epipleoneura haroldoi Santos, 1964 & MPU; MAO; NAI; PRF & $3 ; 100 ; 63 ; 103$ \\
\hline Epipleoneura humeralis (Selys, 1886) & $\mathrm{COA}$ & $3 ; 99 ; 63 ; 103$ \\
\hline Epipleoneura kaxuriana Machado, 1985 & MPU; MAO; NRP; PRF; RPE; NAI & $3 ; 100 ; 63 ; 103$ \\
\hline Epipleoneura lamina Williamson, 1915 & BOR & 63 \\
\hline Epipleoneura manauensis Santos, 1964 & BAR; MPU; MAO; RPE; NAI & $3 ; 95 ; 100 ; 98 ; 63 ; 57 ; 96 ; 103$ \\
\hline Epipleoneura metallica Rácenis, 1955 & MAO & 97 \\
\hline Homeoura obrieni von Ellenrieder, 2008 & MAO & 88 \\
\hline Hylaeonympha magoi Rácenis, 1968 & AMZ & 2 \\
\hline Inpabasis machadoi Santos, 1961 & MPU; MAO; NAI & $3 ; 2 ; 100 ; 76 ; 46 ; 103$ \\
\hline Inpabasis rosea (Selys, 1877) & MPU; MAO & $3 ; 95 ; 2 ; 100 ; 76 ; 46 ; 103$ \\
\hline Ischnura capreolus (Hagen, 1861) & MPU; MAO; SGC & $3 ; 95 ; 57 ; 97 ; 103$ \\
\hline Ischnura fluviatilis Selys, 1876 & MAO; PRF & $3 ; 91 ; 103$ \\
\hline Leucobasis candicans Rácenis, 1959 & AMZ & 2 \\
\hline Mecistogaster linearis (Fabricius, 1777) & BAR; MAO; NAI; PRF & $3 ; 72 ; 103$ \\
\hline Mecistogaster lucretia (Drury, 1773) & MAO; PRF & $3 ; 100 ; 97 ; 103$ \\
\hline Mesoleptobasis acuminata Santos, 1961 & IRN; CAR & $95 ; 100 ; 46$ \\
\hline Mesoleptobasis cantralli Santos, 1961 & BAR; SGC & $3 ; 2 ; 100 ; 98 ; 32 ; 103$ \\
\hline $\begin{array}{l}\text { Mesoleptobasis elongata Garrison \& von } \\
\text { Ellenrieder, } 2009\end{array}$ & CAR & $2 ; 32$ \\
\hline Mesoleptobasis incus Sjöstedt, 1918 & MPU; NAI; BOR; TEF & $3 ; 2 ; 100 ; 98 ; 99 ; 32 ; 46 ; 103$ \\
\hline Metaleptobasis amazonica Sjöstedt, 1918 & MAO & $1 ; 32 ; 46$ \\
\hline Metaleptobasis brysonima Williamson, 1915 & PRF & $2 ; 90$ \\
\hline
\end{tabular}


Continuation...

Metaleptobasis paludicola von Ellenrieder, 2013

Metaleptobasis tridentigera von Ellenrieder, 2013

Microstigma anomalum Rambur, 1842

Microstigma maculatum Hagen in Selys, 1860

Microstigma rotundatum Selys, 1860

Neoneura bilinearis Selys, 1860

Neoneura desana Machado, 1989

Neoneura luzmarina De Marmels, 1989

Neoneura mariana Williamson, 1917

Neoneura rufithorax Selys, 1886

Neoneura sylvatica Hagen in Selys, 1886

Oxyagrion terminale Selys, 1876

Phasmoneura exigua (Selys, 1886)

Phoenicagrion flammeum (Selys, 1876)

Platystigma buckleyi (McLachlan, 1881)

Protoneura scintilla Gloyd, 1939

Protoneura tenuis Selys, 1860

Psaironeura bifurcata (Sjöstedt, 1918)

Psaironeura tenuissima (Selys, 1886)

Telebasis abuna Bick \& Bick, 1995

Telebasis dunklei Bick \& Bick, 1995

Telebasis griffinii (Martin, 1896)

Telebasis inalata (Calvert, 1961)

Telebasis obsoleta (Selys, 1876)

Telebasis sanguinalis Calvert, 1909

Telebasis simulata Tennessen, 2002

Tuberculobasis inversa (Selys, 1876)

Tukanobasis corbeti Machado, 2009

ANISOPTERA

Aeshnidae

Anax amazili (Burmeister, 1839)

Anax concolor Brauer, 1865

Castoraeschna tepuica De Marmels, 1989

Coryphaeschna adnexa (Hagen, 1861)

Coryphaeschna amazonica De Marmels, 1989

Coryphaeschna viriditas Calvert, 1952

Gynacantha auricularis Martin, 1909

Gynacantha dryadula Neiss \& De Marmels, 2017

Gynacantha gracilis (Burmeister, 1839)

Gynacantha klagesi Williamson, 1923

Gynacantha litoralis Williamson, 1923

Gynacantha membranalis Karsch, 1891

Gynacantha mexicana Selys, 1868

Gynacantha nervosa Rambur, 1842

Gynacantha tenuis Martin, 1909

MAO
NAI; MPU
MAO
MAO; PRF

BAR; MPU; MAO; PRF; SGC; TEF; SPO; NAI

BAR

COA

BAR; NRP; PRF; RPE; SGC; MAO

MAO

COA; ATA

AMZ

AMZ

ITA; MPU; MAO; NAI; PRF; RPE

MAO; NAI

AMZ

BAR

MAO; PRF

MPU; MAO; PRF; NAI

MPU; MAO; PRF; RPE

IRN

IRN; CAR

IRN

AMZ

IRN; CAR

AMZ

PRF; MAO

TEF

SGC
$95 ; 2 ; 90$

2; 90

$95 ; 100 ; 97$

$3 ; 100 ; 61 ; 103$

3; 95; 100; 80; 103

$3 ; 103$

$3 ; 100 ; 98 ; 103$

$3 ; 100 ; 35 ; 103$

95

$3 ; 100 ; 35 ; 50 ; 103$

51

$2 ; 100 ; 4$

$3 ; 95 ; 100 ; 98 ; 57 ; 103$

3; 95; 2; 100; 98; 103

$100 ; 49$

$3 ; 103$

$3 ; 103$

$3 ; 100 ; 36 ; 97 ; 103$

3; 95; 100; 99; 103

95

95

$95 ; 2$

2

$95 ; 2 ; 38 ; 46$

$100 ; 46$

$3 ; 95 ; 2 ; 100 ; 38 ; 46 ; 103$

2; 99; 52

2; 53 
Koroiva R. et al.

Continuation...

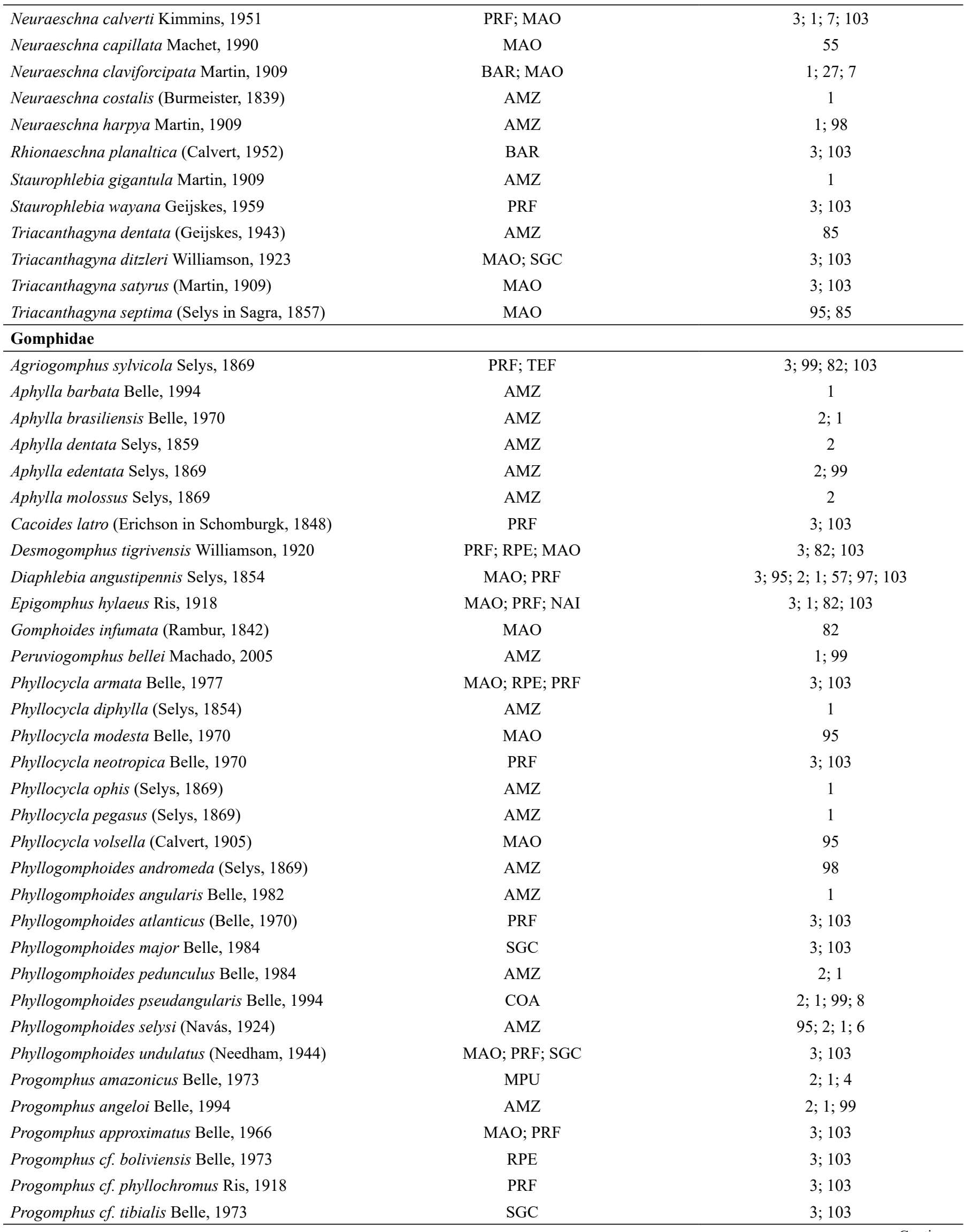


Continuation...

Progomphus delicatus Belle, 1973

Progomphus fassli Belle, 1973

Progomphus guyanensis Belle, 1966

Progomphus intricatus Hagen in Selys, 1858

Progomphus maculatus Belle, 1984

Progomphus perpusillus Ris, 1918

Progomphus pijpersi Belle, 1966

Zonophora batesi Selys, 1869

Zonophora calippus Selys, 1869

Zonophora nobilis Belle, 1983

Zonophora supratriangularis Schmidt, 1941

Zonophora wucherpfennigi Schmidt, 1941

$\begin{array}{cc}\text { NAI; PRF } & 3 ; 103 \\ \text { AMZ } & 1 \\ \text { MAO; PRF } & 3 ; 103 \\ \text { AMZ } & 2 \\ \text { MAO } & 3 ; 103 \\ \text { AMZ } & 2 ; 1 ; 4 \\ \text { AMZ } & 2 \\ \text { AMZ } & 2 ; 1 ; 99 \\ \text { TEF } & 2 ; 1 ; 99 ; 5 \\ \text { SGC } & 2 ; 1 ; 5 \\ \text { MAO; TEF } & 95 ; 2 ; 1 ; 99 ; 5 \\ \text { MAO; PRF; RPE } & 3 ; 2 ; 1 ; 99 ; 103\end{array}$

SPO

$56 ; 66$

\begin{tabular}{l} 
Neocordulia batesi (Selys, 1871) \\
\hline Corduliidae \\
\hline Aeschnosoma auripennis Geijskes, 1970
\end{tabular}

Aeschnosoma forcipula Hagen in Selys, 1871

Aeschnosoma hamadae Fleck \& Neiss, 2012

, MAO; PRF; NAI; BAR

$66 ; 3 ; 2 ; 62 ; 21 ; 103$

BAR; MPU; MAO; NAI; PRF

MAO; PRF; NAI

$3 ; 95 ; 2 ; 1 ; 41 ; 103$

$3 ; 2 ; 29 ; 103$

BAR

$2 ; 29 ; 3 ; 103$

Paracordulia sericea (Selys, 1871)

MAO

65

\begin{tabular}{l}
\hline Lauromacromia cf. dubitalis (Fraser, 1939) \\
\hline Libellulidae \\
\hline Anatya guttata (Hoffmansegg in Schomburgk, \\
1848)
\end{tabular}

Argyrothemis argentea Ris, 1911

Brachymesia herbida (Gundlach, 1889)

Brechmorhoga nubecula (Rambur, 1842)

Brechmorhoga praedatrix Calvert, 1909

Dasythemis esmeralda Ris, 1910

Dasythemis essequiba Ris, 1919

Diastatops estherae Montgomery, 1940

Diastatops maxima Montgomery, 1940

Diastatops nigra Montgomery, 1940

Diastatops obscura (Fabricius, 1775)

Diastatops pullata (Burmeister, 1839)

Elasmothemis williamsoni (Ris, 1919)

Elga leptostyla Ris, 1911

Erythemis attala (Selys in Sagra, 1857)

Erythemis carmelita Williamson, 1923

Erythemis haematogastra (Burmeister, 1839)

Erythemis mithroides (Brauer, 1900)

Erythemis peruviana (Rambur, 1842)

Erythemis plebeja (Burmeister, 1839)

Erythemis vesiculosa (Fabricius, 1775)

Erythrodiplax amazonica Sjöstedt, 1918

Erythrodiplax anatoidea Borror, 1942
COA

MAO; MPU; NAI

MAO; PRF

BAR; MAO; PRF

PRF

AMZ

BAR; MAO; MAU; SGC; TON

PRF; TEF

BAR; MAO; MAU; PAR; PRF; SGC

MAO

BAR; PRF; MAU; MAO; MPU; SPO

MAO; PRF

NAI; MAO; NRP; PRF

NAI; PRF; MAO; BEC; PAR; SPO

BEC; TEF

MAO; BAR; PRF; PAR; MNE; BEC

PAR

IRN; NRP; MAO; PAR; ITA

MAO

IRN; MAO; NAI; PRF; MNE

BAR; PRF; MAO; MAO

MAO; PRF
PRF; RPE; MAO

\section{0}

$3 ; 16$

$95 ; 98 ; 91 ; 21 ; 15$

$3 ; 103$

$3 ; 103$

$3 ; 95 ; 57 ; 103$

1

$3 ; 1 ; 45 ; 58 ; 101 ; 103$

$1 ; 99 ; 58 ; 101$

$3 ; 1 ; 98 ; 58 ; 101 ; 103$

102

$3 ; 1 ; 98 ; 99 ; 58 ; 103$

$3 ; 103$

$3 ; 103$

$3 ; 95 ; 2 ; 99 ; 73 ; 15 ; 68 ; 97 ; 103$

$1 ; 94 ; 68$

$3 ; 95 ; 21 ; 91 ; 73 ; 15 ; 68 ; 103$

73

$3 ; 95 ; 98 ; 45 ; 73 ; 15 ; 68 ; 103$

73

$3 ; 21 ; 91 ; 73 ; 15 ; 68 ; 103$

$3 ; 95 ; 2 ; 1 ; 14 ; 103$

$3 ; 103$ 
Continuation..

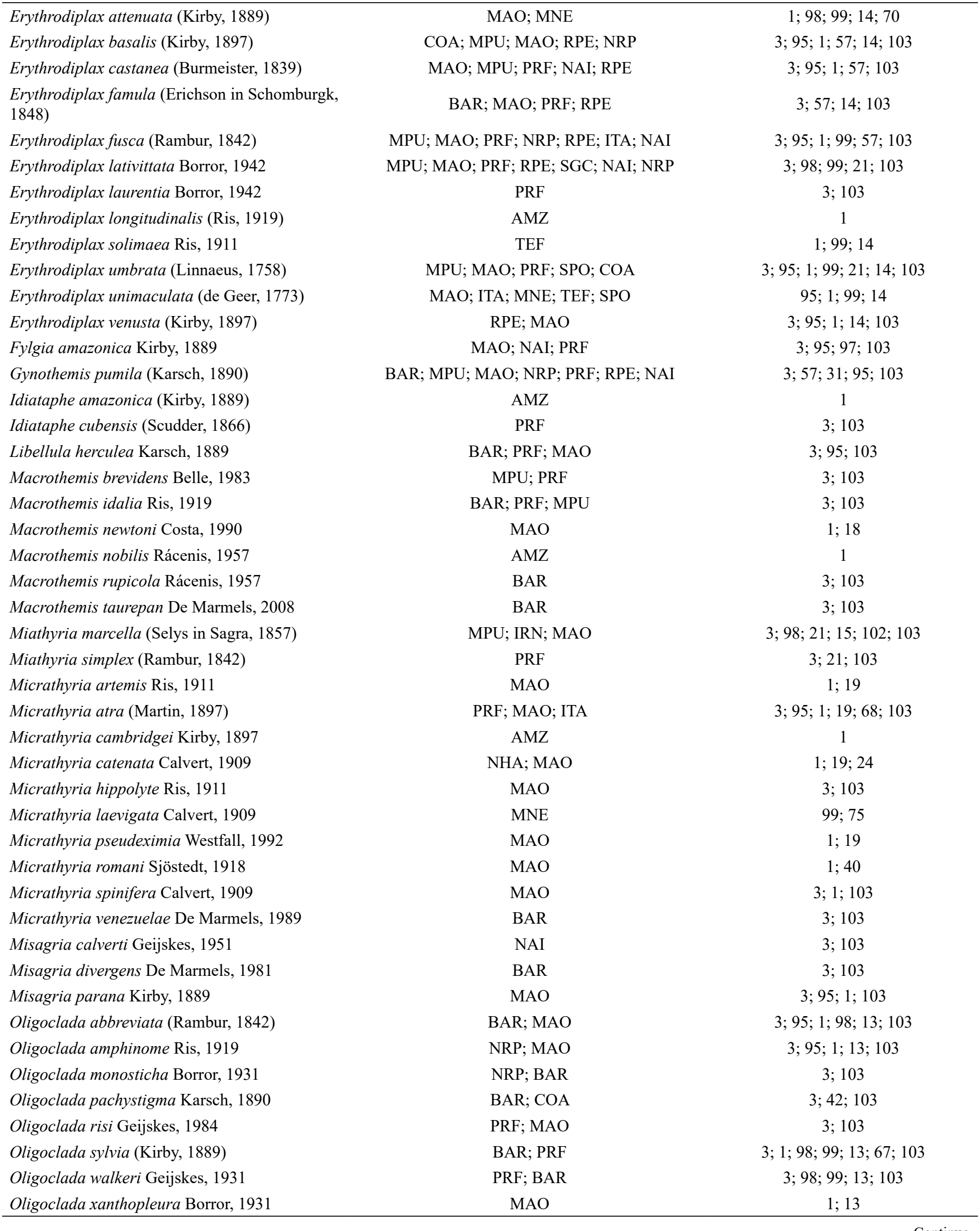

Continue... 
Continuation...

Orthemis aequilibris Calvert, 1909

Orthemis attenuata (Erichson in Schomburgk, 1848)

Orthemis biolleyi Calvert, 1906

Orthemis cultriformis Calvert, 1899

Orthemis discolor (Burmeister, 1839)

Orthemis ferruginea (Fabricius, 1775)

Pantala flavescens (Fabricius, 1798)

Perithemis bella Kirby, 1889

Perithemis cornelia Ris, 1910

Perithemis electra Ris, 1930

Perithemis lais (Perty, 1834)

Perithemis mooma Kirby, 1889

Perithemis thais Kirby, 1889

Rhodopygia cardinalis (Erichson in Schomburgk, 1848)

Rhodopygia geijskesi Belle, 1964

Rhodopygia hollandi Calvert, 1907

Tauriphila australis (Hagen, 1867)

Tholymis citrina Hagen, 1867

Tramea binotata (Rambur, 1842)

Tramea calverti Muttkowski, 1910

Tramea minuta De Marmels \& Rácenis, 1982

Tramea rustica De Marmels \& Rácenis, 1982

Uracis fastigiata (Burmeister, 1839)

Uracis imbuta (Burmeister, 1839)

Uracis infumata (Rambur, 1842)

Uracis ovipositrix Calvert, 1909

Uracis siemensi Kirby, 1897

Zenithoptera anceps Pujol-Luz, 1993

Zenithoptera fasciata (Linnaeus, 1758)

Zenithoptera lanei Santos, 1941

Zenithoptera viola Ris, 1910
MPU; MAO

RPE

MAO; NRP; PRF; RPE

$\mathrm{SPO}$

MPU; MAO; RPE; NAI

MAO

MPU; MAO; PRF

MAO; SPO

TEF

BEC

BAR; MAO; SGC

MAO

BAR; PRF; BEC

MAO

MAO; SAI

AMZ

MAO

BAR; ITA; MAO; NRP; PRF; MNE

BAR

RPE; ITA; PRF; MAO; TEF

BAR; SPO

MAO

PRF; SPO; MAO; MNE; JUR; GJR; EIR; BEC; TAB

MAO; NRP; CAR; ATA; MNE; SPO; BEC; TAB; SGC

PRF; MAO; SPO; EIR

NAI; MAO; SPO; MNE; SGC

PRF; BAR; MAO; NRP; PAR; MNE; EIR; SPO

MAO; ITA

MPU; PRF; MAO; BOR; ITA; PAR

NAI; PRF; ITA; MAO; TAB

ITA
$3 ; 89 ; 103$

$3 ; 103$

$3 ; 57 ; 102 ; 103$

$1 ; 77$

$3 ; 103$

$15 ; 57$

$3 ; 2 ; 21 ; 57 ; 103$

3; $1 ; 102 ; 103$

$1 ; 99 ; 71$

79

$3 ; 2 ; 1 ; 98 ; 99 ; 91 ; 71 ; 103$

$3 ; 103$

$3 ; 1 ; 81 ; 103$

$1 ; 68$;

$9 ; 68$

1

3; 98; 103

$3 ; 95 ; 103$

$3 ; 103$

$3 ; 95 ; 2 ; 1 ; 99 ; 21 ; 22 ; 103$

$3 ; 2 ; 1 ; 22 ; 103$

$2 ; 22$

$3 ; 1 ; 98 ; 99 ; 70 ; 17 ; 103$

$3 ; 95 ; 1 ; 98 ; 99 ; 57 ; 70 ; 17 ; 103$

$3 ; 1 ; 17 ; 103$

3; 95; 1; 98; 99; 70; 17; 103

$3 ; 95 ; 1 ; 99 ; 17 ; 103$

$2 ; 69$

$3 ; 2 ; 57 ; 95 ; 69 ; 102 ; 103$

$3 ; 2 ; 98 ; 69 ; 103$

$95 ; 2$
Diastatops intensa Montgomery, 1940 and Diastatops dimidiata Montgomery, 1940 were registered for the floodplains of the SolimõesAmazonas rivers by Nessimian et al. (2008). Although most of their sampled sites were in the Amazonas state, there are some sites in Pará state as well, and authors do not present a detailed list of where each species was found.

Despite the significant increase in the number of species known from Amazonas, some regions remain poorly explored, such as municipalities in the southwest region of the state. Besides Guajará (code number 10) and Eirunepé (code number 12), there are no published species records from municipalities located on the border with Acre state. In addition, large municipalities also appear to be under-sampled. For example, São Gabriel da Cachoeira, the third largest municipality in Brazil $\left(109,181 \mathrm{~km}^{2}\right.$; IBGE 2019) with an area larger than Portugal, only has 20 recorded species. In this way, we suggest that new field expeditions should focus on such large municipalities and the 29 municipalities that presented no specimen records in our survey.

In general, knowledge about the Odonata order in Amazonas state has improved, especially in the last two decades. However, limitations on sampling still underestimate the number of odonates species, both for new species and new records. Therefore, this work recognizes the importance of the biological diversity in Amazonas state and the Amazonian Biome for species richness of Brazil and shows that we still do not entirely know the odonatofauna in the world's largest tropical forest. 

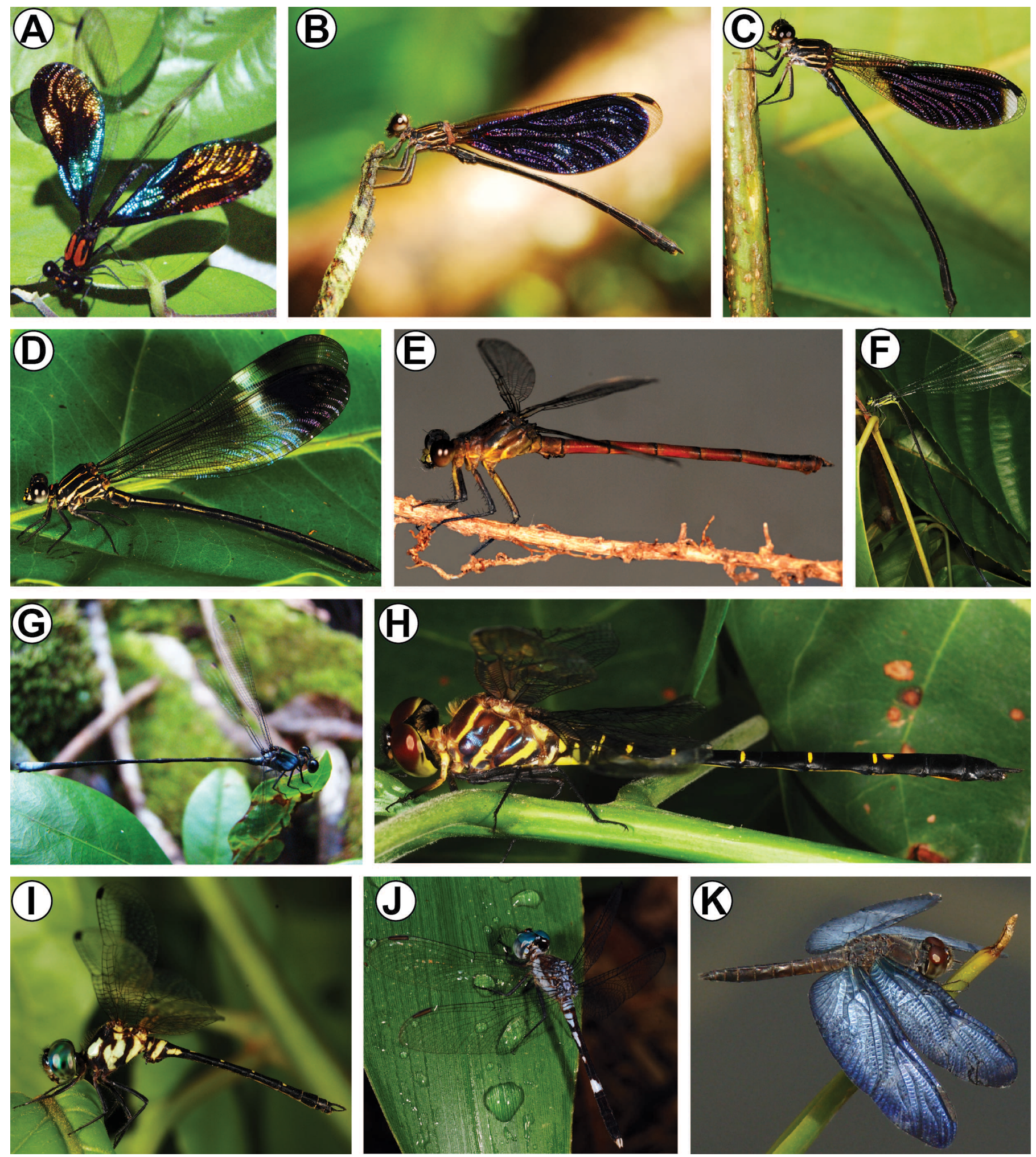

Figure 2. Some Odonata species recorded in Amazonas State, Brazil. A, Chalcopteryx rutilans (Rambur 1842); B, Chalcopteryx scintillans McLachlan, 1870; C, Chalcopteryx seabrai Santos \& Machado, 1961; D, Polythore vittata (Selys, 1869); E, Rimanella arcana (Needham, 1933); F, Mecistogaster linearis (Fabricius, 1777); G, Dimeragrion percubitale Calvert, 1913; H, Aeschnosoma hamadae Fleck \& Neiss, 2012; I, Elga leptostyla Ris, 1911; J, Erythrodiplax anatoidea Borror, 1942; K, Zenithoptera lanei Santos, 1941. 


\section{Acknowledgements}

We are thankful to FAPEAM (Fundação de Amparo à Pesquisa do Estado do Amazonas) for a post-doctoral fellowship (FIXAM-I/ FAPEAM; Proc.062.01503/2018) to RK. NH is a CNPq research fellow (Proc. 307849/2014-7). INPA/ MCTI and PRONEX-CNPq-FAPEAM provided partial financial support for this research.

\section{Author Contributions}

Ricardo Koroiva: Contribution to data collection and manuscript preparation; Substantial contribution in the concept and design of the study; Contribution to critical revision, adding intellectual content.

Ulisses Gaspar Neiss: Contribution to data collection and manuscript preparation; Substantial contribution in the concept and design of the study; Contribution to critical revision, adding intellectual content.

Günther Fleck: Contribution to manuscript preparation; Contribution to critical revision, adding intellectual content.

Neusa Hamada: Contribution to data collection and manuscript preparation; Contribution to critical revision, adding intellectual content.

\section{Conflicts of Interest}

The authors declare that they have no conflict of interest related to the publication of this manuscript.

\section{References}

ABBOTT, J.C. 2015. Dragonflies of Texas: A Field Guide. University of Texas Press.

ALVARES, C.A., STAPE, J.L., SENTElHAS, P.C., DE MORAES GONÇALVES, J.L. \& SPAROVEK, G. 2013. Köppen's climate classification map for Brazil. Meteorol. Zeitschrift 22(6):711-728.

BELLE, J. 1973. A revision of the New World genus Progomphus Selys, 1854 (Anisoptera: Gomphidae). Odonatologica 2:191-308.

BELLE, J. 1983. A review of the genus ZonophoraSelys (Odonata, Gomphidae). Tijdschr. voor Entomol. 126(7/8):145-173.

BELLE, J. 1984. A synopsis of the South American species of Phyllogomphoides, with a key and descriptions of three new taxa (Odonata, Gomphidae). Tijdschr. voor Entomol. 127(4):79-100.

BELLE, J. 1989. A revision of the New World genus Neuraeschna Hagen, 1867 (Odonata: Aeshnidae). Tijdschr. voor Entomol. 132(2):259-284.

BELLE, J. 1994. On five species of Phyllogomphoides Belle, 1970, from Brazil with the descriptions of three new taxa (Odonata: Gomphidae). Zool. Meded. 68:73-85.

BELLE, J. 1998. Synopsis of the Neotropical genus Rhodopygia Kirby, 1889 (Odonata: Libellulidae). Zool. Meded. 72(1):1-13.

BICK, G.H. \& BICK, J.C. 1985. A revision of the picta group of Polythore, with a description of a new species, P. lamerceda spec. nov., from Peru (Zygoptera: Polythoridae). Odonatologica 14(1):1-28.

BICK, G.H. \& BICK, J.C. 1986. The genus Polythore exclusive of the picta group (Zygoptera: Polythoridae). Odonatologica 15(3):245-273.

BICK, G.H. \& BICK, J.C. 1988. A review of males of the genus Philogenia, with descriptions of five new species from South America (Zygoptera: Megapodrionidae). Odonatologica 17:9-32.

BORROR, D.J. 1931. The genus Oligoclada (Odonata). Misc. Publ. Museum Zool. Univ. Michigan 22:1-64.

BORROR, D.J. 1942. A revision of the Libelluline genus Erythrodiplax (Odonata). The Ohio state university, Columbus.

CALVERT, P.P. 1908. Odonata. In Biologia Centrali-Americana p.17-420.
COSTA, J.M. 1990. Macrothemis newtoni spec. nov., a new dragonfly from Brazil (Anisoptera: Libellulidae). Odonatologica 19:75-79.

COSTA, J.M., LOURENÇO, A.N. \& VIEIRA, L.P. 2002. Micrathyria pseudhypodidyma sp. n. (Odonata: Libellulidae), com Chave das Espécies do Gênero que Ocorrem no Estado do Rio de Janeiro. Neotrop. Entomol. 31(3):377-389.

COSTA, J.M., MACHADO, A.B.M., LENCIONI, F.A.A. \& SANTOS, T.C. 2000. Diversidade e distribuição dos Odonata (Insecta) no estado de São Paulo, Brasil: Parte I - Lista das espécies e registros bibliográficos. Publicaçoes Avulsas do Mus. Nac. 80:1-27.

COSTA, J.M. \& OLDRINI, B.B. 2003. Descrição da fềmea de Argyrothemis argentea (Odonata, Libellulidae). Iheringia. Série Zool. 93(3):271-276.

COSTA, J.M. \& OLDRINI, B.B. 2005. Diversidade e distribuição dos Odonata (Insecta) no estado do Espírito Santo, Brasil. Publicaçoes Avulsas do Mus. Nac. 107:1-15.

COSTA, J.M. \& SANTOS, T.C. 1997. Intra- and interspecific variation in the genus Uracis Rambur, 1842, with a key to the known species (Anisoptera: Libellulidae). Odonatologica 261-7.

COSTA, J.M. \& SANTOS, T.C. 2000. Espécie nova de Heteragrion Selys, 1862 do estado do Rio de Janeiro, Brasil (Odonata: Zygoptera: Megapodagrionidae). Bol. do Mus. Nac. do Rio Janeiro / Zool. (411):1-7.

DALZOCHIO, M.S., RENNER, S., SGANZERLA, C., PRASS, G., ELY, G.J., SALVI, L.C., DAMETTO, N. \& PÉRICO, E. 2018. Checklist of Odonata (Insecta) in the state of Rio Grande do Sul, Brazil with seven new records. Biota Neotrop. 18(4):e20180551. DOI: 10.1590/1676-0611-bn-2018-0551

DE MARCO, P. 1998. The Amazonian campina dragonfly Assemblage: Patterns in microhabitat use and behaviour in foraging habitat (Anisoptera). Odonatologica 27:239-248.

DE MARCO-JÚNIOR, P., NÓBREGA, C.C., DE SOUZA, R.A. \& NEISS, U.G. 2015. Modeling the distribution of a rare Amazonian odonate in relation to future deforestation. Freshw. Sci. 34(3):1123-1132.

DE MARMELS, J. 1987. On the type specimens of some neotropical Megapodagrionidae, with a description of Heteragrion pemon spec. nov. and Oxystigma caerulans spec. nov. from Venezuela (Zygoptera). Odonatologica 16(3):225-238.

DE MARMELS, J. 1989. Odonata or dragonflies from Cerro de la Neblina and the adjacent lowland between the Rio Baria, the Casiquiare and Rio Negro (Venezuela) I Adultos. Bol. la Acad. Ciencias Fis. Mat. y Nat. 25:1-78.

DE MARMELS, J. 2001. Revision of Megapodagrion Selys, 1886 (Insecta, Odonata: Megapodagrionidae). Ph.D. thesis, Universität Zürich.

DE MARMELS, J. \& NEISS, U.G. 2011. Description of the larva of Gynacantha auricularis Martin, 1909 (Odonata: Aeshnidae). Zootaxa 3137:64-88.

DE MARMELS, J. \& NEISS, U.G. 2013. Description of the larva of Neuraeschna claviforcipata Martin, 1909. (Insecta: Odonata: Aeshnidae). Zootaxa 3721:97-100

DE MARMELS, J. \& RÁCENIS, J. 1982. An analysis of the cophysa-group of Tramea Hagen, with descriptions of two new species (Anisoptera: Libellulidae). Odonatologica 11(2):109-128.

DIJKSTRA, K.-D.B., BECHLY, G., BYBEE, S.M., DOW, R.A., DUMONT, H.J., FLECK, G., GARRISON, R.W., HÄMÄLÄINEN, M., KALKMAN, V.J., KARUBE, H., MAY, M.L., ORR, A.G., PAULSON, D.R., REHN, A.C., THEISCHINGER, G., TRUEMAN, J.W.H., VAN TOL, J., VON ELLENRIEDER, N. \& WARE, J. 2013. The classification and diversity of dragonflies and damselflies (Odonata). In: Zhang, Z.-Q. (Ed.) Animal Biodiversity: An Outline of Higher-level classification and survey of taxonomic richness. Zootaxa 3703(1):36.

DIJKSTRA, K.D.B., KALKMAN, V.J., DOW, R.A., STOKVIS, F.R. \& VAN TOL, J. 2014. Redefining the damselfly families: A comprehensive molecular phylogeny of Zygoptera (Odonata). Syst. Entomol. 39(1):68-96.

DUNKLE, S.W. 1991. Review of the neotropical damselfly family Dicteriadidae (new spelling), with an annotated bibliography (Zygoptera). Odonatologica 20(4):401-416.

FEARNSIDE, P.M. 2019. Retrocessos sob o Presidente Bolsonaro: Um Desafio à Sustentabilidade na Amazônia. Sustentabilidade Int. Sci. J. 1(1):38-52. 
FLECK, G. 2018. Oxygastra Selys, 1870 et genres apparentés, un ancien groupe enraciné dans le Crétacé. Rev. Sci. Bourgogne-Franche-Comté Nat. 27:295-301.

FLECK, G. \& NEISS, U.G. 2012. A new species of the genus Aeschnosoma Selys, 1870 (Odonata: Anisoptera: Corduliidae s. s.). Zootaxa 3159:47-58.

FULAN, J.Â.,ANJOS, M.R. dos \& MACHADO, N.G. 2015. Effect of temperature on the life cycle of Acanthagrion Selys, 1876 (Insecta: Coenagrionidae) under artificial conditions. Biota Neotrop. 15(2):e20140169.

GARRISON, R.W. 1990. A synopsis of the genus Hetaerina with descriptions of four new species (Odonata: Calopterygidae). Trans. Am. Entomol. Soc. 116(1):175-259.

GARRISON, R.W. 1999. The genus Neoneura, with keys and description of a new species, Neoneura jurzitzai spec. nov. (Zygoptera: Protoneuridae). Odonatologica 28(4):343-375.

GARRISON, R.W. 2004. An analysis of the Psaironeura tenuissima complex, including synonymy of P. machadoi De Marmels with P. bifurcata Sjöstedt (Odonata: Protoneuridae). Odonatologica 33(1):83-89.

GARRISON, R.W. 2006. A synopsis of the genera Mnesarete Cowley, Bryoplathanon gen. nov., and Ormenophlebia gen. nov. (Odonata: Calopterygidae). Contrib. to Sci. Nat. Hist. Museum Los Angeles Cty. 50:61-84.

GARRISON, R.W. 2009. A synopsis of the genus Telebasis Selys (Odonata: Coenagrionidae). Int. J. Odonatol. 12:1-121.

GARRISON, R.W. 2014. Review of Oxystigma Selys with the synonymy of Oxystigma williamsoni Geijskes (Odonata: Heteragrionidae). Zootaxa 3780(2):347-364.

GARRISON, R.W. \& ELLENRIEDER, N. von. 2015. Damselflies of the genus Agria of the Guiana Shield (Odonata: Coenagrionidae). Zootaxa 4042(1):1-134.

GARRISON, R.W. \& ELLENRIEDER, N. Von. 2006. Generic diagnoses within a confusing group of genera: Brechmorhoga, Gynothemis, Macrothemis, and Scapanea (Odonata: Libellulidae). Can. Entomol. 138:269-284.

GARRISON, R.W. \& ELLENRIEDER, N. Von. 2009. Redefinition of Mesoleptobasis Sjöstedt 1918 with the inclusion of Metaleptobasis cyanolineata (Wasscher 1998) comb. nov. and description of a new species, Mesoleptobasis elongata (Odonata: Coenagrionidae). Zootaxa 2145:47-68.

GEIJSKES, D.C. 1963. Three new species of Micrathyria. Stud. Fauna Suriname other Guyanas 17:70-81.

GEIJSKES, D.C. 1970. Generic characters of the South American Corduliidae, with descriptions of the species found in the Guyanas. Stud. Fauna Suriname other Guyanas 12:1-42.

GEIJSKES, D.C. 1984. What is Oligoclada abbreviata (Rambur, 1842)? (Odonata: Libellulidae). Zool. Meded. 58(12):175-185.

GUILLERMO-FERREIRA, R., NEISS, U.G., HAMADA, N. \& BISPO, P.C. 2014. Behavior of the Amazonian damselfly Chalcopteryx scintillans McLachlan (Zygoptera: Polythoridae) and comments on its morphological distinction from C. rutilans (Rambur). Int. J. Odonatol. 17(4):251-258.

HAMADA, N. \& OLIVEIRA, S.J. 2003. Food items of larvae of Rimanella arcana (Needham, 1933) (Odonata: Amphipterygidae) in Central Amazonia, Brazil. Entomotropica 18(2):153-155.

HECKMAN, C.W. 2006. Encyclopedia of South American Aquatic Insects: Odonata - Anisoptera. Springer Netherlands, Dordrecht.

HECKMAN, C.W. 2008. Encyclopedia of South American Aquatic Insects: Odonata - Zygoptera. Springer Netherlands, Dordrecht.

IBGE. 2019. Cidades e estados. https://cidades.ibge.gov.br/ (last access on 04/08/2019).

JUEN, L. 2011. Grandes rios e a distribuição de Odonata na Amazônia: similaridade de composição, limitação a dispersão e endemismo. Ph.D. thesis, Universidade Federal de Goiás.

JUEN, L. \& DE MARCO, P. 2011. Odonate biodiversity in terra-firme forest streamlets in Central Amazonia: on the relative effects of neutral and niche drivers at small geographical extents. Insect Conserv. Divers. 4(4):265-274.

KIRBY, W.F. 1897. LXII.- - List of the Neuroptera collected by Mr. E. E. Austen on the Amazons \&c. during the recent Expedition of Messrs. Siemens Bros. Cable S.S. 'Faraday,' with descriptions of several new species of Odonata (Dragonflies). Ann. Mag. Nat. Hist. 19(114):598-617.
KOROIVA, R., RODRIGUES, M.E., VALENTE-NETO, F. \& ROQUE, F. de O. 2017. Odonates from Bodoquena Plateau: checklist and information about endangered species. Biota Neotrop. 17(3):e20160310. DOI: 10.1590/16760611-bn-2016-0310.

LENCIONI, F.A.A. 2005. Damselflies of Brazil: An Illustrated Identification Guide, Volume 1: Non-Coenagrionidae Families. All Print Editora, São Paulo, Brazil.

LENCIONI, F.A.A. 2006. Damselflies of Brazil: An Illustrated Identification Guide, Volume 2: Coenagrionidae. All Print Editora, São Paulo, Brazil.

LENCIONI, F.A.A. 2013. Diagnoses and discussion of the group 1 and 2 Brazilian species of Heteragrion, with descriptions of four new species (Odonata: Megapodagrionidae). Zootaxa 3685(1):1-80.

LEONARD, J.W. 1977. A revisionary study of the genus Acanthagrion (Odonata: Zygoptera). Misc. Publ. Museum Zool. Univ. Michigan 153:1-173.

MACHADO, A.B.M. 2002. Studies on neotropical Protoneuridae. 13: The types of Neoneura rufithorax Selys (Zygoptera). Not. Odonatol. 5(9):115-116.

MACHADO, A.B.M. 2007. Studies on neotropical Protoneuridae. 20. Neoneura kiautai spec. nov. from Southeastern Brazil (Zygoptera, Protoneuridae). In Odonata: Biology of Dragonflies (B. K. Tyagi, ed.) Scientific Publishers, Jodhpur, p.25-32.

MACHADO, A.B.M. 2009a. Denticulobasis and Tuberculobasis, new genera close to Leptobasis, with description of ten new species (Odonata: Coenagrionidae). Zootaxa 1208:1-36.

MACHADO, A.B.M. 2009b. Tukanobasis gen. nov. with the description of T. corbeti sp. nov. from the Amazonian region of Brazil (Odonata: Coenagrionidae). Int. J. Odonatol. 12(2):331-336.

MACHADO, A.B.M. 2012. The apicale species group of Acanthagrion with description of four new species and a hook- moving apparatus (Zygoptera: Coenagrionidae). Odonatologica 41(3):201-223.

MACHADO, A.B.M. \& LACERDA, D.S.S. 2017. Revalidation of Platystigma Kennedy, 1920, with a synopsis of the quadratum species group and the description of three new species (Odonata: Pseudostigmatidae). Zootaxa 4242(3):493.

MACHET, P. 1990. Deux nouvelles espèces d'Aeshnidae de la Guyane française: Neuraeschna clavulata et Neuraeschna capillata (Odonata: Anisoptera). Entomologiste 46(5):209-218.

MAY, M. 1991. A review of the genus Neocordulia, with a description of Mesocordulia subgen. nov. and of Neocordulia griphus spec. nov. from Central America, and a note on Lauromacromia (Odonata: Corduliidae). Folia Entomológica Mex. 82:17-67.

MONTEIRO-JÚNIOR, C.S., COUCEIRO, S.R.M., HAMADA, N. \& JUEN, L. 2013. Effect of vegetation removal for road building on richness and composition of Odonata communities in Amazonia, Brazil. Int. J. Odonatol. 17:1-13.

MONTGOMERY, B.E. 1940. A revision of the genus Diastatops (Libellulidae, Odonata) and a study of the leg characters of related genera. Lloydia $3: 213-280$.

NEISS, U.G. 2012. Taxonomia de Odonata (Insecta), com ênfase na caracterização morfológica e biologia de larvas, na Amazônia Central, Brasil. Ph.D. thesis, Instituto Nacional de Pesquisa da Amazônia (INPA).

NEISS, U.G., FLECK, G., FEITOZA, L.A.M. \& HAMADA, N. 2013. Description of the adult male of Aeschnosoma auripennis Geijskes, 1970 (Odonata: Corduliidae s.s.). Zootaxa 3718(6):596.

NEISS, U.G. \& HAMADA, N. 2010. The larva of Perilestes attenuatus Selys, 1886 (Odonata: Perilestidae) from Amazonas, Brazil. Zootaxa 2614:53-58.

NEISS, U.G. \& HAMADA, N. 2012. Larvae of Epipleoneura manauensis Santos and Roppaneura beckeri Santos with a key to the genera of known Neotropical Protoneuridae larvae (Odonata: Zygoptera). Int. J. Odonatol. 15(1):31-43.

NEISS, U.G. \& HAMADA, N. 2014. Ordem Odonata. In Insetos aquáticos na Amazônia brasileira : taxonomia, biologia e ecologia (N. Hamada, J. L. Nessimian, \& R. B. Querino, eds) Editora do INPA, Manaus, p.217-282.

NEISS, U.G. \& HAMADA, N. 2016. Larva of Palaemnema brasiliensis Machado (Odonata: Platystictidae), from Amazonas, Brazil. Zootaxa 4078(1):70-77. 
NEISS, U.G., LENCIONI, F.A.A., HAMADA, N. \& FERREIRA-KEPPLER, R.L. 2008. Larval redescription of Microstigma maculatum Hagen in Selys, 1860 (Odonata: Pseudostigmatidae) from Manaus, Amazonas, Brasil. Zootaxa 169657-62.

NEISS, U.G. \& DE MARMELS, J. 2017 Gynacantha dryadula sp. nov. from the Guiana Shield (Odonata, Anisoptera: Aeshnidae). Zootaxa 4254(5): 563-574.

NESSIMIAN, J.L., HAMADA, N., FERREIRA-JR., N. \& MARCO-JR, P. de. 2008. Entomofauna Aquática. In Conservação da Várzea: Identificação e Caracterização de Regiões Biogeográficas (A. L. K. M. Albernaz, ed.) Ibama/Pro-Várzea, Manaus, p.93-131.

NÓBREGA, C.C. \& DE MARCO, P. 2011. Unprotecting the rare species: A niche-based gap analysis for odonates in a core Cerrado area. Divers. Distrib. 17(3):491-505.

OLAYA, M. 2019. Odonatos en Latinoamérica: la riqueza de nuestra región. Hetaerina 1(2):4-5.

PARÉDIO, J.L. 2012. Ordenamento territorial: uma análise das politicas territoriais no Estado do Amazonas. Master dissertation. Universidade Federal do Amazonas.

PESSACQ, P. 2014. Synopsis of Epipleoneura (Zygoptera, Coenagrionidae, "Protoneuridae"), with emphasis on its Brazilian species. Zootaxa 3872(3):201-234.

PESSACQ, P., SANTOS, T.C. \& COSTA, J.M. 2012. Checklist and updated distribution of Protoneuridae from Brazil. Int. J. Odonatol. 15(2):59-73.

PINTO, Â.P. 2013. Análise cladística de Sympetrinae Tillyard, 1917 com ênfase no grupo de armadura femoral especializada: os gêneros de "Erythemismorpha" (Insecta: Odonata: Libellulidae). Ph.D. thesis. Universidade de São Paulo, São Paulo.

PINTO, A.P. 2019. Odonata in Catálogo Taxonômico da Fauna do Brasil. PNUD. http://fauna.jbrj.gov.br/fauna/listaBrasil/ConsultaPublicaUC/ BemVindoConsultaPublicaConsultar.do?invalidatePageControlCounter $=5 \&$ lingua $=\& j$ sonRank $=\% 5 \mathrm{~B} \% 7 \mathrm{~B}+\% 22 \mathrm{rank} \% 22 \% 3 \mathrm{~A} \% 22 \mathrm{ORDEM} \% 22 \% 2 \mathrm{C}+$ $\% 22$ nome $\% 22 \% 3 \mathrm{~A} \% 22 \mathrm{Odonata} \% 22+\% 7 \mathrm{D}+\% 5 \mathrm{D} \&$ rankTaxon $=32767 \&$ no meTaxon $=\&$ nomeTaxon $=$ Odonata\&autor $=\&$ nomeVernaculo $=\&$ nomeCompl eto $=\&$ formaVida $=$ QUALQUER $\&$ substrato $=$ QUALQUER\&ocorrencia $=\mathrm{OC}$ ORRE\&regiao $=$ QUALQUER\&estado $=$ QUALQUER\&endemismo $=$ TODO $\mathrm{S} \&$ origem $=$ TODOS\&mostrarAte $=$ SUB_ESPECIE\&opcoesBusca $=$ NOME ACEITO (last access on 12/04/2019)

PINTO, A.P. \& CARVALHO, A.L. 2010. A new species of Lauromacromia (Odonata: Corduliidae) from Southeastern Brazil, with a cladistic analysis of the genus and comments on Neotropical dragonfly biogeography. Zootaxa 2425:45-68.

PINTO, Â.P. \& LAMAS, C.J.E. 2010. Navicordulia aemulatrix sp. nov. (Odonata, Corduliidae) from northeastern Santa Catarina State, Brazil. Rev. Bras. Entomol. 54(4):608-617.

PINTO, A.P. \& LAMAS, C.J.E. 2011. Oligoclada mortis sp. nov. from Rondônia State, Brazil, and distributional records of other species of the genus (Odonata: Libellulidae). Int. J. Odonatol. 14(4):291-303.

PIRES, M.M., KOTZIAN, C. B., SGANZERLA, C., PRASS, G., DALZOCHIO, M.S. \& PÉRICO, E. 2019. Diversity of Odonata (Insecta) in Seasonal Deciduous Forest fragments in southern Brazil (state of Rio Grande do Sul), with a new record for the state and comments on the seasonal distribution of the species. Biota Neotrop. 19(4): e20190769. DOI: 10.1590/1676-0611bn-2019-0769.

PUJOL-LUZ, J.R. \& FONSECA, R.R. 1997. Wings colour variation and geographical distribution of the genus Zenithoptera Bates (Odonata, Libellulidae). Rev. da Univ. Rural - Série Ciências da Vida 19(1-2):13-26.

ST. QUENTIN, D. 1973. Die Gomphidenfauna Südamerikas (Ordn.: Odonata). Ann. des Naturhistorischen Museums Wien 77:335-363.

RIS, F. 1910. Libellulinen 3. Collect. Zool. du Baron Edm. Selys Longchamps 11:245-384.

RIS, F. 1911. Libellulinen 4. Collect. Zool. du Baron Edm. Selys Longchamps 12:385-528.

RIS, F. 1930. A revision of the Libelluline genus Perithemis (Odonata). Misc. Publ. Univ. Michigan Museum Zool. 21(21):1-50.
ROCHEDO, P.R.R., SOARES-FILHO, B., SCHAEFFER, R., VIOLA, E., SZKLO, A., LUCENA, A.F.P., KOBERLE, A., DAVIS, J.L., RAJÃO, R. \& RATHMANN, R. 2018. The threat of political bargaining to climate mitigation in Brazil. Nat. Clim. Chang. 8(8):695-698.

RODRIGUES, M.E., KOROIVA, R., RAGALZI-DA-SILVA, E. \& DE MOURA, E.B. 2016. Mecistogaster linearis (Fabricius) (Odonata: Coenagrionidae): First Record from Mato Grosso do Sul State, Brazil. EntomoBrasilis 9(3):212-215.

RODRIGUES, M.E., MOURA, E.B., KOROIVA, R., BORGES, A.C.P. \& ROQUE, F. de O. 2018. Survey of Dragonflies (Odonata) in Palm Swamps of Cerrado Hotspot. Entomol. News 128(1):24-38.

RODRÍGUEZ, F.P., SARMIENTO, C.E. \& GONZÁLEZ-SORIANO, E. 2015. Morphological variability and evaluation of taxonomic characters in the genus Erythemis Hagen, 1861 (Odonata: Libellulidae: Sympetrinae). Insecta mundi 428:1-68.

SANTOS, N.D. 1949. "Planiplax machadoi" n. sp. e notas sobre outras espécies (Odonata, Libellulidae). Rev. Bras. Biol. 9(4):427-432.

SANTOS, N.D. 1954. Revisão de Micrathyria didyma (Selys, 1857) e suas correlatas. (Libellulidae: Odonata). Arch. do Mus. Nac. (Rio Janeiro) 42:491-498.

SANTOS, N.D. 1961. Inpabasis g.n. e três espécies novas (Coenagriidae: Odonata). Bol. Mus. Para. Emilio Goeldi 34:1-7.

SANTOS, N.D. 1967. Contribuição ao conhecimento da fauna do Estado da Guanabara, 58.-Notas sobre "Orthemis cultriformis" Calvert, 1899 (Odonata, Libellulidae). Atas da Soc. Biol. do Rio Janeiro 11127-128.

SANTOS, N.D. 1970a. Contribuição ao conhecimento da fauna do Estado da Guanabara. 73. Notas sobre a ninfa, o imago e a emergência de Coryphaeschna adnexa (Hagen, 1861) Calvert, 1903 (Odonata, Aehnidae). Atas da Soc. Biol. do Rio Janeiro 13:75-77.

SANTOS, N.D. 1970b. Contribuição ao conhecimento da fauna do Estado da Guanabara. 76 - Descrição da ninfa de Perithemis electra Ris, 1930 e notas sobre o macho (Odonata: Libellulidae). Atas da Soc. Biol. do Rio Janeiro 14(3/4):49-50.

SANTOS, N.D. \& MACHADO, A.B. 1960. Contribuição ao conhecimento do gêero Chalcopteryx Selys, 1853, com a descrição de uma nova espécie. Bol. do Mus. Para. Emilio Goeldi (24):1-17.

SCHMIDT, E. 1952. Odonata nebst Bemerkungen über die Anomisma und Chalcopteryx des Amazonas-Gebiets. In Beiträge zur Fauna Perus nach der Ausbeute der Hamburger Südperu Expedition (E. Titschack, ed.) p.207-256.

SILVEIRA, L.F., BEISIEGEL, B. de M., CURCIO, F.F., VALDUJO, P.H., DIXO, M., VERDADE, V.K., MATTOX, G.M.T. \& CUNNINGHAM, P.T.M. 2010. Para que servem os inventários de fauna? Estud. Avançados 24(68):173-207.

SPINDOLA, L. de A., SOUZA, L.O.I. \& COSTA, J.M. 2001. Descrição da larva de Perithemis thais Kirby, 1889, com chave para identificação das larvas das espécies conhecidas do gênero citadas para o Brasil (Odonata: Libellulidae). Bol. Do Mus. Nac. Nov. Ser. Zool. 442:1-8.

TENNESSEN, K.J. 2015. Four new species of Calvertagrion St. Quentin from South America (Odonata: Coenagrionidae). Odonatologica 44(3):397-430.

TORREIAS, S.R. da S., NEISS, U.G., HAMADA, N., FERREIRA-KEPPLER, R.L. \& LENCIONI, F.A.A. 2008. Description of the larva of Bromeliagrion rehni (Odonata: Coenagrionidae) with bionomic notes concerning its phytotelmic habitat in central Amazonas, Brazil. Rev. Bras. Zool. 25(3):479-486.

VILAÇA, Z.A.S. 2017. Distribuição espacial da riqueza de Odonata (Fabricius, 1793) em relação às ecorregiões neotropicais: determinantes ambientais e restrições à dispersão. Master dissertation. Universidade Federal de Goiás.

VILELA, D.S., GUILLERMO-FERREIRA, R. \& CORDERO-RIVERA, A. 2018. Description of the female of Dicterias atrosanguinea Selys 1853, with notes on male genital ligula and male behavior (Odonata: Dicteriadidae). Zootaxa 4374(3):441-450.

VILELA, D.S.; CORDERO-RIVERA, A. \& GUILLERMO-FERREIRA, R. 2019. The female of Heteragrion icterops Selys, 1862 (Odonata: Heteragrionidae) with comments and key on the Brazilian females of Heteragrion group B. Zootaxa 4576(1): 187-194. 
VILELA, D.S., KOROIVA, R., TOSTA, T.H.A., NOVAES, M.C. \& GUILLERMO-FERREIRA, R. 2020. Dragonflies and damselflies from the West of Minas Gerais, Brazil: checklist and new records. Biota Neotrop. 20(1): e20190851. DOI: 10.1590/1676-0611-BN-2019-0851

VON ELLENRIEDER, N. 2008. Revalidation of Argentagrion and redefinition of Homeoura, with the description of H. obrieni n. sp. (Odonata: Coenagrionidae). Rev. la Soc. Entomológica Argentina 67(1/2):81-106.

VON ELLENRIEDER, N. 2009. Five new species of Orthemis from South America (Odonata: Libellulidae). Int. J. Odonatol. 12:347-381.

VON ELLENRIEDER, N. 2013. A revision of Metaleptobasis Calvert (Odonata: Coenagrionidae) with seven synonymies and the description of eighteen new species from South America. Zootaxa 3738(1):1-155.

VON ELLENRIEDER, N. \& GARRISON, R.W. 2003. A synopsis of the genus Triacanthagyna (Odonata: Aeshnidae). Int. J. Odonatol. 6(2):147-184.

VON ELLENRIEDER, N. \& GARRISON, R.W. 2008. The genus Oligoclada in Argentina, with description of $\mathrm{O}$. rubribasalis sp nov (Odonata : Libellulidae). Int. J. Odonatol. 11(2):249-260.
WALKER, I. 2009. Emergence of aquatic insects and spider abundance in the Balbina Reservoir (Presidente Figueiredo, Amazonas, Brazil) during the phase of declining eutrophication. Acta Limnol. Bras. 21(2):199-207.

WILLIAMSON, E.B. 1923. Notes on the genus Erythemis with a description of a new species (Odonata). Misc. Publ. Museum Zool. Univ. Michigan 11:1-18.

WILLIAMSON, E.B. \& WILLIAMSON, J.H. 1924a. A remarkable new genus of Coenagrionidae from Brazil, with descriptions of three new species (Odonata). Occas. Pap. Museum Zool. Univ. Michigan 154:1-24.

WILLIAMSON, E.B. \& WILLIAMSON, J.H. 1924b. The genus Perilestes (Odonata). Misc. Publ. Museum Zool. Univ. Michigan 14:1-39.

Received: $28 / 08 / 2019$

Revised: 05/12/2019

Accepted: 09/12/2019

Published online: 31/01/2020 